\title{
Endovascular Treatment of a Huge Hepatic Artery Aneurysm by Coil Embolization Method:A Case Report
}

\author{
Hossein Hemmati, ${ }^{1,}$ Mehdi Karimian, ${ }^{1}$ Habibollah Moradi, ${ }^{1}$ Kambiz Farid Marandi, ${ }^{1}$ and \\ Afrooz Haghdoost ${ }^{2}$ \\ ${ }_{1}^{1}$ Respiratory Diseases and TB Research Center, Razi Hospital, Guilan University of Medical Sciences, Rasht, Iran \\ ${ }^{2}$ Student Research Committee, Department of Research and Technology, Guilan University of Medical Sciences, Rasht, Iran \\ ${ }^{*}$ Corresponding author: Hossein Hemmati, Respiratory Diseases and TB Research Center, Razi Hospital, Guilan University of Medical Sciences, Rasht, Iran. Tel: +98-9133045924, Fax: \\ +98-1333542460, E-mail: drkarimianm@yahoo.com
}

Received: April 23, 2012; Revised: June 23, 2013; Accepted: November 30, 2013

\begin{abstract}
Hepatic artery aneurysms are rare but potentially life threatening. We describe a novel case of a successful endovascular coil embolization of a huge hepatic artery aneurysm. A 67-year-old woman presented with recent abdominal pain that had begun from 2 weeks before referring to our hospital. Sonographic and computerized tomographic (CT) findings revealed a huge hepatic artery aneurysm with 95 $\mathrm{mm} \times 83 \mathrm{~mm}$ diameter. The patient underwent an endovascular technique. In aortic angiography, the celiac artery orifice and superior mesenteric artery were so narrow, so sonography was used in order to determine the exact position of the catheter in the celiac artery orifice. The aneurysm was thrombosed using coil embolization. Pulsation of the aneurysm immediately disappeared. Huge hepatic artery aneurysm can be safely treated using coil embolization.
\end{abstract}

Keywords: Hepatic Artery; Aneurysm; Endovascular Procedures; Pain; Embolization; Therapeutic

\section{Introduction}

Visceral artery aneurysms (VAAs) are rare lesions among other vascular diseases with an incidence of 0.01 - $2 \%$, although the thorough incidence is not known because of the asymptomatic nature of VAAs (1). Because of their high risk for rupture (30 - 40\% of cases), which is an emergent and potentially life threatening situation, they can cause high morbidity and mortality. Small VAAs have no symptoms (2), but large ones can have symptoms such as pain, bleeding and hypotension (3) and they occur usually in splenic, hepatic, superior mesenteric, gastroduodenal and small pancreatic arteries (4).

The first case of hepatic artery aneurysm (HAA) was reported in 1809 in a dead patient (5). Some studies reported a $20 \%$ incidence for HAAs that can occur intrahepatic (because of inflammation and trauma) or extrahepatic (because of atherosclerosis) $(6,7)$. HAAs are the second most frequent aneurysms after splenic artery aneurysm and their rupture is life threatening $(8,9)$.

In the past, many VAAs were diagnosed after rupture (1), but in the recent decades, advances in rapid cross-sectional body imaging, ultrasonography, magnetic resonance imaging (MRI) and computed tomography (CT) has enabled us to identify them earlier $(1,2,6)$. The gold standard for diagnosis and pre-operative planning is digital subtraction angiography (5).

Repair is recommended in aneurysms greater than $2 \mathrm{~cm}$ in diameter (5). Anatomic suitability, clinical presentation, underlying etiology, general health status and comorbidity factors determine the treatment method. Before using endovascular techniques, for many years, open surgical treatment was the only treatment method $(10,11)$.

At present, endovascular techniques provide an alternative method in which good results with low morbidity and recurrence rates have been reported (11). In cases of intracranial aneurysm, embolization with coils has been increasingly used for treatment. Studies showed that it is a safe method for patients with an unruptured aneurysm or aneurysmal subarachnoid hemorrhage (12).

Herein, we present a patient with a rare huge hepatic artery aneurysm ( $95 \mathrm{~mm} \times 83 \mathrm{~mm}$ diameter) that caused recent abdominal pain and abdominal mass. She was treated successfully with endovascular coil embolization (with as many as five coils).

\section{Case Presentation}

A 67-year-old woman was admitted for recent right upper abdominal pain that began 2 weeks ago. The pain was non positional, sustained and unrelated to eating. There were no other abdominal symptoms. The patient had no significant past medical history. During the initial physical examination, there was a pulsatile large mass in the right

Copyright (C) 2015, Tehran University of Medical Sciences and Iranian Society of Radiology. This is an open-access article distributed under the terms of the Creative Commons Attribution-NonCommercial 4.0 International License (http://creativecommons.org/licenses/by-nc/4.0/) which permits copy and redistribute the material just in noncommercial usages, provided the original work is properly cited. 
upper quadrant of the abdomen. In duplex ultrasound, a huge arterial aneurysm was detected in the hepatic artery with mural thrombosis. Computerized tomogram (CT) with contrast medium showed a huge aneurysm $(95 \mathrm{~mm}$ $\times 83 \mathrm{~mm}$ in diameter) in the hepatic artery that contained mural thrombi in the aneurysmal sac (Figure 1).

The patient underwent endovascular technique. Transfemoral celiac artery catheterization was not successful so the trunk was catheterized via the left brachial artery approach. In aortic angiography, the celiac artery orifice and superior mesenteric artery were narrow too. Sonography was used to determine the exact position of the catheter in the celiac artery orifice. Then selective hepatic artery catheterization was done. The tip of the catheter (JR4 6F) was placed in the hepatic artery distal to the gastroduodenal artery. Three coils (MR eye Embolization coil $8 \mathrm{~mm} \times 5$ $\mathrm{cm}$ ) were delivered in the aneurysm and two others were delivered in the hepatic artery (neck of aneurysm). The aneurysm began to thrombose and eventually thrombosed completely after 4 to 5 minutes (Figure 2). The patient was uneventful after the procedure and pulsation of the mass disappeared. Two days after the procedure, ultrasound showed complete thrombosis of the aneurysm (Figure 3 ). Liver function tests were normal after the procedure and 3 days after that. The patient was discharged with good general condition.

\section{Discussion}

HAA is an uncommon vascular disease (13). Approximately $20 \%$ of all visceral aneurysms are HAAs (6). They usually have no symptoms (5) but some patients with HAA have abdominal pain (55\%), gastrointestinal hemorrhage or hemobilia (up to $46 \%$ ), and obstructive jaundice (7). Our patient has a huge hepatic artery aneurysm. Atherosclerosis, vasculitis, fibromuscular dysplasia and

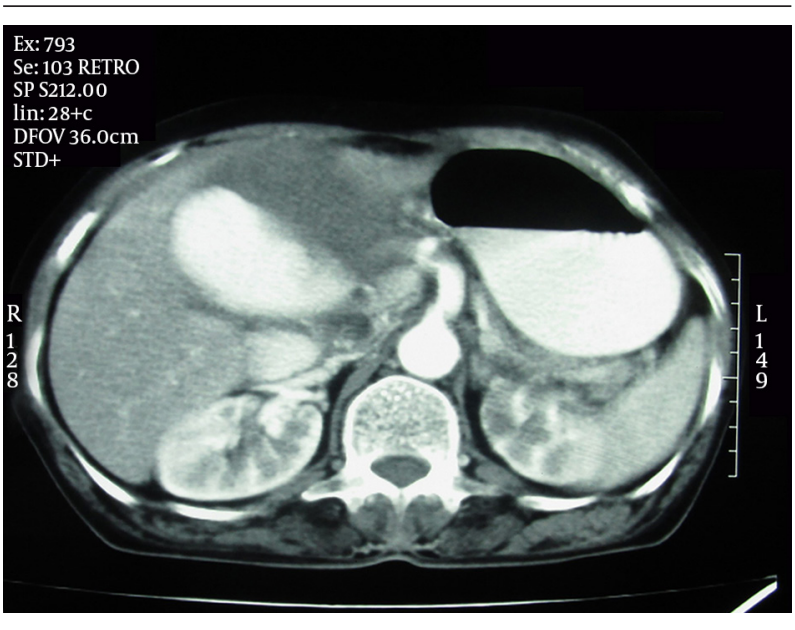

Figure 1. A 67-year-old woman referred with abdominal pain. Computer ized tomogram (CT) of the abdomen with IV and oral contrast reveals a huge aneurysm ( $95 \mathrm{~mm} \times 83 \mathrm{~mm}$ diameter) in the hepatic artery containing mural thrombosis in the aneurysmal sac.
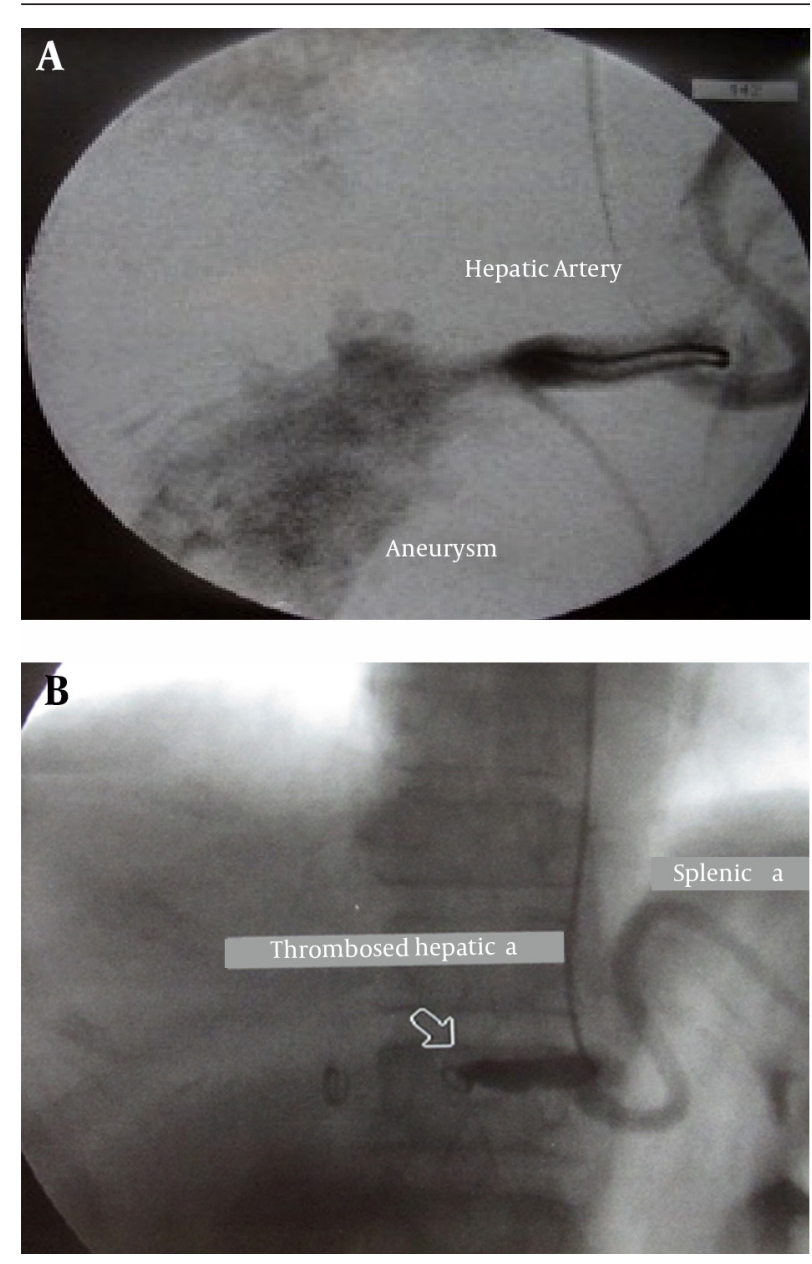

Figure 2. The digital subtraction angiogram of hepatic artery aneurysm before (A) and after (B) coil embolization shows complete thrombosis of the aneurysm.

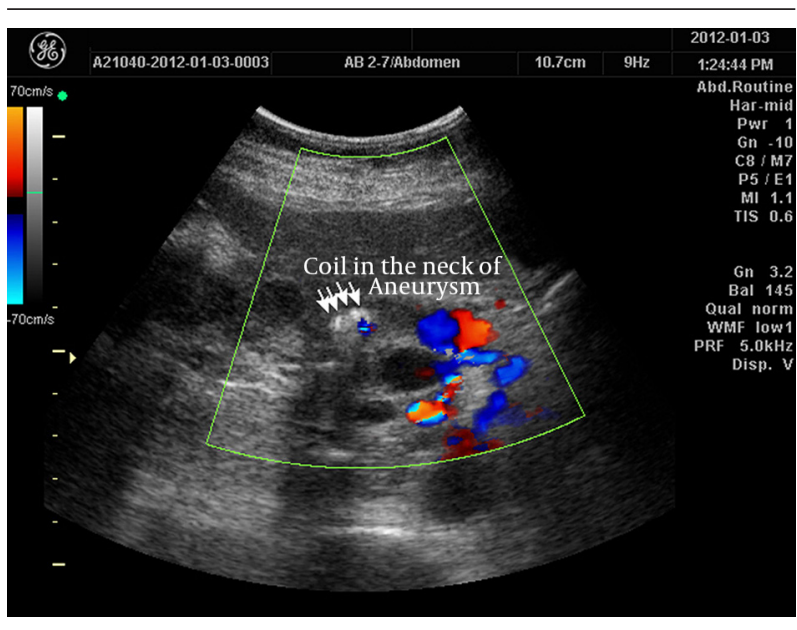

Figure 3. Ultrasonography two days after coil embolization shows complete thrombosis of the aneurysm. 
cystic medial necrosis are common causes for HHAs (7). Selective angiography of the celiac axis and superior mesenteric artery, CT scan and ultrasound are diagnostic methods for this aneurysm $(3,14)$. Open surgical repair or follow up of the patients with serial imaging are traditional methods for managingVAAs (10), but some studies reported a $30 \%$ to $50 \%$ mortality rate for the surgical treatment $(5,6)$. Nowadays, we can use various methods for VAA treatment such as mentioned conventional open surgery, laparoscopic surgery, and endovascular treatment (3). Endovascular (or percutaneous) approach uses different methods and instruments such as coil, cyanoacrylate, thrombin and stent graft (3). Endovascular treatment may have better outcomes in both elective and emergency cases because it is less invasive (15). Site and morphology of VAAs determine the feasibility of embolization technique for the patient (3). In cases with difficult anatomy, coil embolization is used due to its relative simplicity (15). In our patient with a huge hepatic artery aneurysm that was high risk for surgery, endovascular coil embolization was selected. In this patient, five coils were used for embolization of the hepatic artery aneurysm. According to this case report and the procedure applied, it seems that coil embolization is a safe and effective procedure to treat hepatic artery aneurysm even in huge sizes.

\section{References}

1. Chiesa R, Astore D, Guzzo G, Frigerio S, Tshomba Y, Castellano R, et al. Visceral artery aneurysms. Ann Vasc Surg. 2005;19(1):42-8.

2. Tulsyan N, Kashyap VS, Greenberg RK, Sarac TP, Clair DG, Pierce $\mathrm{G}$, et al. The endovascular management of visceral artery aneurysms and pseudoaneurysms. J Vasc Surg. 2007;45(2):276-83.

3. Lagana D, Carrafiello G, Mangini M, Dionigi G, Caronno R, Cas- telli P, et al. Multimodal approach to endovascular treatment of visceral artery aneurysms and pseudoaneurysms. Eur J Radiol. 2006;59(1):104-11.

4. Gabelmann A, Gorich J, Merkle EM. Endovascular treatment of visceral artery aneurysms. J Endovasc Ther. 2002;9(1):38-47.

5. de Ruiter-Derksen GL, Bruijnen RC, Joosten F, Reijnen MM. Endovascular treatment of a hepatic artery aneurysm causing chronic abdominal pain; a case report. Ann Hepatol. 2010;9(1):104-6.

6. Abbas MA, Fowl RJ, Stone WM, Panneton JM, Oldenburg WA, Bower TC, et al. Hepatic artery aneurysm: factors that predict complications. J Vasc Surg. 2003;38(1):41-5.

7. O'Driscoll D, Olliff SP, Olliff JF. Hepatic artery aneurysm. Br J Radiol. 1999;72(862):1018-25.

8. Rothbarth LJ, Redmond PL, Kumpe DA. Percutaneous transhepatic treatment of a large intrahepatic aneurysm. AJR Am J Roentgenol.1989;153(5):1077-8.

9. Venturini M, Angeli E, Salvioni M, De Cobelli F, Trentin C, Carlucci $\mathrm{M}$, et al. Hemorrhage from a right hepatic artery pseudoaneurysm: endovascular treatment with a coronary stent-graft. J Endovasc Ther. 2002;9(2):221-4.

10. Saltzberg SS, Maldonado TS, Lamparello PJ, Cayne NS, Nalbandian MM, Rosen RJ, et al. Is endovascular therapy the preferred treatment for all visceral artery aneurysms? Ann Vasc Surg. 2005;19(4):507-15.

11. Sessa C, Tinelli G, Porcu P, Aubert A, Thony F, Magne JL. Treatment of visceral artery aneurysms: description of a retrospective series of 42 aneurysms in 34 patients. Ann Vasc Surg. 2004;18(6):695-703.

12. Brilstra EH, Rinkel GJ, van der Graaf Y, van Rooij WJ, Algra A. Treatment of intracranial aneurysms by embolization with coils: a systematic review. Stroke. 1999;30(2):470-6.

13. Harlaftis NN, Akin JT. Hemobilia from ruptured hepatic artery aneurysm. Report of a case and review of the literature. Am J Surg. 1977;133(2):229-32.

14. Harvey J, Dardik H, Impeduglia T, Woo D, DeBernardis F. Endovascular management of hepatic artery pseudoaneurysm hemorrhage complicating pancreaticoduodenectomy. J Vasc Surg. 2006;43(3):613-7.

15. Pulli R, Dorigo W, Troisi N, Pratesi G, Innocenti AA, Pratesi C. Surgical treatment of visceral artery aneurysms: A 25-year experience. J Vasc Surg. 2008;48(2):334-42. 